\title{
Fruto-oligossacarídeos: aspectos \\ nutricionais, tecnológicos e sensoriais
}

\section{Fructooligosaccharides: nutritional, technological and sensory aspects}

\author{
Leandro Levate Macedo ${ }^{1 *}$ (D), Wallaf Costa Vimercati ${ }^{1}$, Cintia da Silva Araújo ${ }^{1}$ \\ ${ }^{1}$ Universidade Federal de Lavras (UFLA), Programa de Pós-graduação em Ciência dos Alimentos, Lavras/MG - \\ Brasil
}

${ }^{*}$ Corresponding Author: Leandro Levate Macedo, Universidade Federal de Lavras (UFLA), Programa de Pós-graduação em Ciência dos Alimentos, Aquenta Sol, Caixa Postal: 3037, CEP: 37200-900, Lavras/MG Brasil, e-mail: leandrolevate@hotmail.com

Cite as: Macedo, L. L., Vimercati, W. C., \& Araújo, C. S. (2020). Fructooligosaccharides: nutritional, technological and sensory aspects. Brazilian Journal of Food Technology, 23, e2019080. https://doi.org/10.1590/1981-6723.08019

\begin{abstract}
Resumo
Este trabalho objetivou apresentar os fundamentos sobre fruto-oligossacarídeos (FOS), seus métodos de obtenção, estabilidade em alimentos processados e aspectos nutricionais. Os FOS são carboidratos naturais presentes em diversas espécies de plantas, compostos por 2 a 10 monômeros de sacarídeos. Além da obtenção natural, que ocorre através da extração em fontes vegetais, esses carboidratos podem ser obtidos pela hidrólise enzimática da inulina, realizada pelas inulinases, ou ainda sintetizados a partir de resíduos de sacarose. Os FOS são estáveis perante a maioria dos processos realizados nos alimentos, sofrendo maiores degradações em processos térmicos envolvendo alta temperatura e/ou pH extremo. Tal característica favorece a industrialização de produtos ricos em FOS e a adição desses compostos como ingredientes. Esse grupo de carboidratos tem ganhado cada vez mais destaque nos últimos anos perante a capacidade de exercerem diversas funções benéficas ao organismo, pois são considerados como prebióticos e fibras solúveis. A ingestão de FOS está associada à redução do risco de câncer de cólon, diabetes, obesidade, doenças cardiovasculares e ao aumento da absorção de alguns minerais. Além disso, são caracterizados pela boa aceitação sensorial, solubilidade e capacidade de retenção de água. Entretanto, o consumo deve ser moderado, pois, em grandes porções, pode causar alguns desconfortos ao indivíduo, tais como flatulência.
\end{abstract}

Palavras-chave: Oligossacarídeos; Prebióticos; Fibra solúvel; Saudabilidade; Alimentos funcionais; Propriedades tecnológicas.

\begin{abstract}
This work aimed to present the fundamentals on fructooligosaccharides (FOS), their methods of production, stability in processed foods and nutritional aspects. FOS are natural carbohydrates present in several species of plants, composed by 2 to 10 monomers of saccharides. In addition to the natural obtaining, which occurs through extraction in vegetable sources, these carbohydrates can be obtained by the enzymatic hydrolysis of inulin, carried out by inulinases, or further synthesized from sucrose residues. The FOS are stable in the majority of food processes, undergoing major degradation in thermal processes involving high temperature and/or extreme $\mathrm{pH}$. This characteristic favors the industrialization of products rich in FOS and the addition of these compounds as
\end{abstract}


ingredients. These groups of carbohydrates have gained more and more prominence in the last years due to the capacity to perform various functions beneficial to the organism, since they are considered as prebiotics and soluble fibers. The intake of FOS is associated with reduced risk of colon cancer, diabetes, obesity, cardiovascular disease, and increased absorption of some minerals. In addition, they are characterized by good sensory acceptance, solubility and water retention capacity. However, consumption should be moderate, because in large portions can cause some discomfort to the individual, such as flatulence.

Keywords: Oligosaccharides; Prebiotics; Soluble fiber; Healthiness; Functional foods; Technological properties.

\section{Introdução}

Os alimentos são matrizes complexas, constituídos por diversas substâncias. Os principais componentes químicos presentes nos alimentos in natura ou processados são carboidratos, proteínas, lipídios e água. Além disso, os alimentos possuem, também, vitaminas, minerais e enzimas. Esses componentes apresentam funções diversas e importantes no nosso organismo, baseadas em atividades estruturais, nutricionais e de reserva (Damodaran et al., 2010).

Os carboidratos são nutrientes básicos e representam a principal fonte de energia da dieta. Encontram-se naturalmente nos alimentos de origem vegetal ou animal, e também podem ser adicionados como ingredientes, durante o processamento. São classificados em monossacarídeos, oligossacarídeos e polissacarídeos (Ordónez et al., 2005; Damodaran et al., 2010; Zakrzewska et al., 2010).

As fibras são carboidratos não digeríveis, podendo ser classificadas segundo as suas características químicas, botânicas e fisiológicas (Costa \& Peluzio, 2012). Nesse grupo, destacam-se os frutanos, que compreendem polímeros naturais formados por frutose, ligada ou não a uma molécula terminal de sacarose. Os frutanos podem ser classificados em levanas [polímeros lineares com ligações glicosídicas $\beta(2 \rightarrow 6)$ ], compostos ramificados [polímeros que possuem ligações tipo $\beta(2 \rightarrow 6)$ e $\beta(2 \rightarrow 1)$ ] e inulina [polímero linear com ligações glicosídicas $\beta(2 \rightarrow 1)$ ]. Os fruto-oligossacarídeos (FOS), por sua vez, pertencem ao grupo da inulina (Roberfroid, 1993; Lorenzoni et al., 2014; Shiomi et al., 2016) e, nos últimos anos, têm despertado grande interesse das indústrias de alimentos dadas suas propriedades tecnológicas e funcionais (Singh et al., 2016).

Diante disso, o objetivo desta revisão é apresentar a definição de FOS, as formas de obtenção, sua estabilidade nos alimentos e os aspectos nutricionais.

\section{Fruto-oligossacarídeos}

\subsection{Definição}

Os oligossacarídeos são compostos por dois a 10 monômeros de sacarídeos. Os fruto-oligossacarídeos (FOS) são oligossacarídeos pertencentes ao grupo dos frutanos, os quais podem ser encontrados na natureza. O termo FOS é utilizado para frutanos do tipo inulina que apresentam ou não um grupo terminal de glicose. $\mathrm{Na}$ composição dos FOS, duas a nove unidades de frutosil estão ligadas através de ligações glicosídicas $\beta(2 \rightarrow 1)$ e a ligação com a glicose é do tipo $\alpha(1 \rightarrow 2)$, como ocorre na sacarose. Os FOS são encontrados, principalmente, nas formas de 1-kestose (GF2), nistose (GF3) e frutofuranosil nistose (GF4) (IUB-IUPAC Joint Commission on Biochemical Nomenclature, 1982; Yun, 1996; Antošová \& Polakovič, 2001; Singh \& Singh, 2010; Vega \& Zuniga-Hansen, 2015).

\subsection{Fontes de Fruto-oligossacarídeos}

Os FOS são compostos de origem natural e podem ser encontrados em mais de 36 mil espécies de plantas, entre as quais destacam-se alcachofra, alho, aspargos, banana, beterraba, cebola, chicória, tomate, trigo e 
yacon (L'Homme et al., 2003; Passos \& Park, 2003; Mussatto \& Mancilha, 2007). Alguns exemplos de alimentos que possuem FOS em sua composição são apresentados na Tabela 1, em que se destaca o yacon.

Tabela 1. Teor de fruto-oligossacarídeos (FOS), em porcentagem de massa fresca, em algumas plantas e vegetais.

\begin{tabular}{cc}
\hline Alimentos & FOS (\%) \\
\hline Alcachofra & $<1$ \\
\hline Alcachofra de Jerusalém & $10-15$ \\
\hline Alho & $3,6-6,4$ \\
\hline Alho-poró & $2,4-8,0$ \\
\hline Aspargos & $2-3$ \\
\hline Banana & $0,3-0,7$ \\
\hline Cebola & $1,1-7,5$ \\
\hline Centeio & $0,5-1$ \\
\hline Cevada & $0,5-1,5$ \\
\hline Chicória & $5-10$ \\
\hline Trigo & $1-4$ \\
\hline Yacon & $3-19$ \\
\hline
\end{tabular}

Fonte: Adaptado de Singh \& Singh (2010) e Alles (2012).

\subsection{Processos de obtenção e produção de FOS}

A obtenção comercial dos FOS pode ocorrer a partir de fontes vegetais, seja por extração direta ou por hidrólise enzimática da inulina. Além disso, um terceiro método consiste na síntese de FOS a partir de atividade enzimática (Voragen, 1998; Antošová \& Polakovič, 2001; Sangeetha et al., 2005).

No primeiro caso, a extração dos FOS é economicamente inviável devido ao baixo rendimento e elevado custo do processo. Desta forma, entre os métodos comerciais, esse é o menos utilizado (Figueroa-González et al., 2011; Singh et al., 2016). Alguns métodos de extração são apresentados no tópico 2.3.1.

No segundo caso, a inulina pode ser extraída por diversos métodos, tais como por difusão em água quente e ultrassom (Lingyun et al., 2007; Li et al., 2015). Para a produção dos FOS, a inulina sofre uma hidrólise enzimática realizada por enzimas inulinases, formando unidades lineares com duas a sete unidades de frutosil com ou sem uma unidade de glicose terminal. As inulinases são classificadas em endo e exoinulinase, podendo ser obtidas a partir de tecidos de plantas e por microrganismos, respectivamente. No entanto, o uso de enzimas oriundas de plantas é limitado devido às condições sazonais e ao baixo rendimento. As endoinulinases hidrolisam aleatoriamente a inulina para a produção de uma mistura de FOS com diferentes graus de polimerização. Já, as exoinulinases hidrolisam a ligação $\beta(2 \rightarrow 1)$ a partir da extremidade não redutora da inulina (Voragen, 1998; Courtin et al., 2009; Singh \& Singh, 2010; Morris \& Morris, 2012; Singh et al., 2016).

$\mathrm{Na}$ terceira situação, os FOS são sintetizados pela transfrutosilação em resíduos de sacarose, realizada pelas enzimas frutosiltransferases ou $\beta$-frutofuranosidases (Blanch et al., 2011), conforme apresentado pela Figura 1. As estruturas dos produtos dessa reação dependem da origem das enzimas utilizadas na síntese. Essas enzimas podem ser produzidas por fungos Aureobasidium pullulans, Aspergillus niger, Aspergillus japonicus, Aspergillus foetidus, Aspergillus oryzae, Aspergillus sydowi, Fusarium oxysporum, Penicillium rugulosum e Scopulariopsis brevicaulis, por levedura Saccharomyces cerevisiae e por bactéria Bacillus macerans (Antošová \& Polakovič, 2001). A $\beta$-frutofuranosidase de Schwanniomyces occidentalis produz 6-kestose em quantidades maiores que 1-kestose. Por sua vez, as $\beta$-frutofuranosidases oriundas de Aspergillus aculeatus, Aspergillus oryzae e Trichoderma reesei produzem apenas 1-kestose, nistose e, em pequenas quantidades, 1-frutofuranosil nistose, todos ligados a uma sacarose por ligação $\beta(2 \rightarrow 1)$ (Nemukula et al., 2009; Vega-Paulino \& Zúniga-Hansen, 2012; Lorenzoni et al., 2014). De modo geral, os FOS obtidos pelo processo de síntese enzimática apresentam entre uma e cinco unidades de frutosil (Passos \& Park, 2003). Esses FOS são produzidos e comercializados como Neosugar $^{\circledR}$, Meioligo ${ }^{\circledR}$, Profeed ${ }^{\circledR}$ e Nutraflora ${ }^{\circledR}$ (Fortes \& Muniz, 2009). 


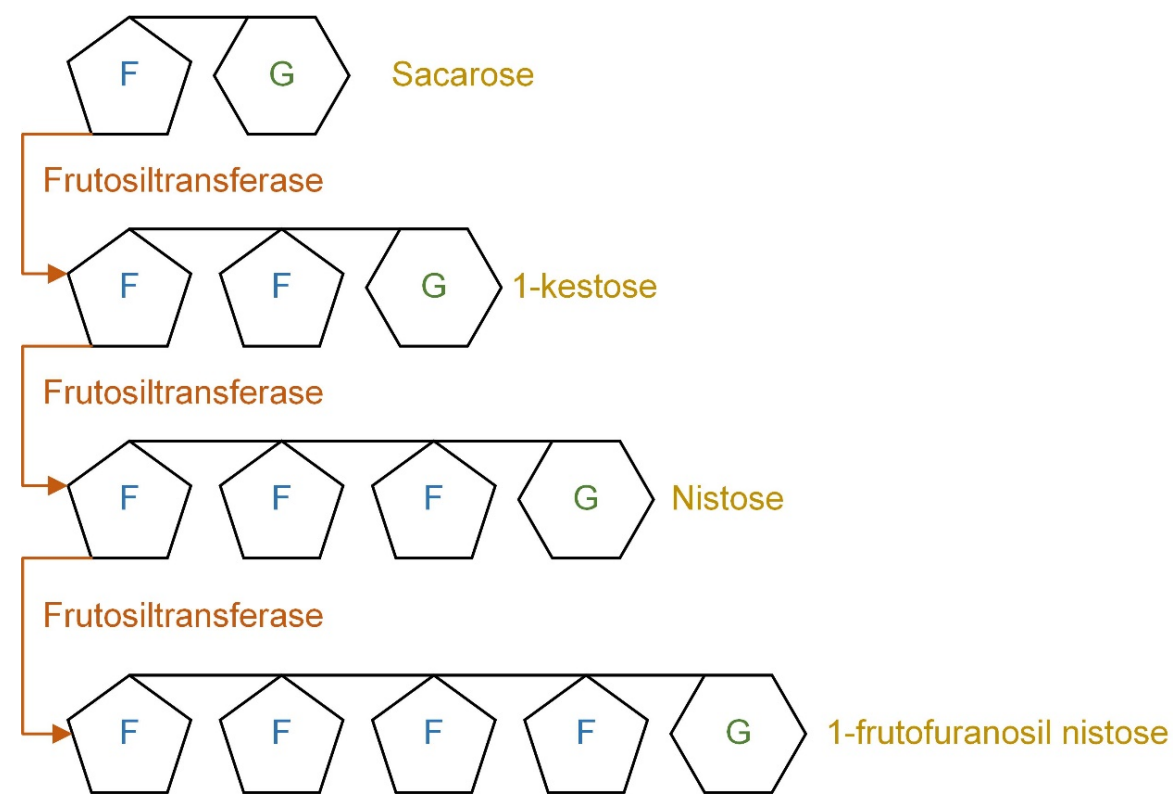

Figura 1. Produção de fruto-oligossacarídeos a partir da sacarose pela frutosiltransferase.

\subsection{Estabilidade dos FOS em alimentos}

Os aspectos relacionados à estabilidade dos FOS são de suma importância, visto que a sua adição em produtos processados pode acarretar a sua degradação e consequente perda do efeito prebiótico.

Os fruto-oligossacarídeos são açúcares não redutores. Esses compostos são estáveis a pH entre 3 e 7, e à temperatura de até $140{ }^{\circ} \mathrm{C}$. A maioria dos processos térmicos, portanto, não é capaz de degradar os FOS. Além disso, por serem não redutores, não são susceptíveis a reação de Maillard e caramelização (Passos \& Park, 2003; Silva et al., 2007; Courtin et al., 2009).

Courtin et al. (2009) avaliaram a estabilidade de diferentes oligossacarídeos ao calor e ao pH. Foi verificado que os FOS foram os mais sensíveis ao meio ácido, sendo que, em $\mathrm{pH} 2$ e 3, ocorreram, respectivamente, 67 e $60 \%$ de hidrólise das ligações glicosídicas $\beta(2 \rightarrow 1)$ após 60 minutos e $100{ }^{\circ} \mathrm{C}$ de incubação. Em pH alcalino, 11, e em temperaturas de 100 e $121{ }^{\circ} \mathrm{C}$, ocorreu decomposição dos FOS. Foi observado, também, que os FOS de cadeia curta apresentaram maior sensibilidade à decomposição alcalina, quando comparados a oligossacarídeos de cadeia longa.

Wang et al. (2009) avaliaram a estabilidade dos FOS durante a pasteurização em temperatura entre 60 e $100{ }^{\circ} \mathrm{C}$ por 30 minutos, e esterilização a $121^{\circ} \mathrm{C}$ por 10 a 50 minutos, em pH de 2 a 4 . Em pH 3,5, foi observado o início da decomposição dos FOS e esses foram completamente hidrolisados em pH 2 e 2,5 na pasteurização. Na esterilização, por sua vez, houve maior degradação de FOS. Em pH abaixo de 3,5, os FOS foram completamente hidrolisados.

Vega \& Zuniga-Hansen (2015) avaliaram a estabilidade de FOS de cadeia curta em tampão de citrato de sódio e sucos de laranja e tomate, em um estudo cinético a pH 3,5. Foi verificado que os pentassacarídeos foram mais estáveis ao tratamento térmico em comparação com os trissacarídeos. Os resultados mostraram que, além da temperatura e do $\mathrm{pH}$, o grau de polimerização, a matriz alimentar e o tipo de pasteurização são parâmetros importantes que devem ser avaliados no processamento de alimentos enriquecidos com FOS. Os FOS foram mais estáveis em suco de laranja, seguido de suco de tomate e tampão de citrato de sódio.

Padma Ishwarya \& Prabhasankar (2013) adicionaram FOS na forma de pó e de xarope em biscoitos. Foi observado que os FOS em pó proporcionaram maior estabilidade e qualidade, visto que apresentaram menor efeito adverso nas características do produto. 
Como verificado nos estudos apresentados acima, o pH exerce grande influência em relação à degradação dos FOS. De acordo com Wang et al. (2009) e Courtin et al. (2009), a degradação de oligossacarideos é fomentada por catálise de prótons e, portanto, favorecida em meio ácido, no qual as ligações frutose-frutose e frutose-glicose tornam-se frágeis, sendo esses compostos rapidamente hidrolisados, quando comparados à degradação em meio básico.

\subsection{Aspectos nutricionais}

O consumo adequado de fibras é importante para a saúde intestinal, contribuindo com a prevenção e o tratamento de diversas patologias, tais como diabetes, doenças cardiovasculares e doenças que acometem o trato gastrointestinal (Kaczmarczyk et al., 2012).

Os FOS são considerados como prebióticos e fibras solúveis, ou seja, são componentes alimentares que não sofrem hidrólise pelas enzimas no trato digestivo, chegando intactos ao intestino grosso. Posteriormente, são fermentados, levando à produção de ácido láctico e ácidos graxos de cadeia curta (AGCC), com consequente diminuição do $\mathrm{pH}$ no intestino grosso. Desta forma, o crescimento de bactérias benéficas ao cólon, como bifidobactérias e lactobacilos, é favorecido. Esses microrganismos são capazes de hidrolisar as ligações dos FOS por possuírem inulinases. A fermentação das fibras solúveis no cólon leva também à formação de gases, tais como $\mathrm{CO}_{2}, \mathrm{H}_{2}$ e $\mathrm{CH}_{4}$. Esses gases, por sua vez, são eliminados na respiração ou podem causar flatulência (Roberfroid, 1993; Gibson \& Roberfroid, 1995; Buddington et al., 2002; Costa \& Peluzio, 2012).

Vaz-Tostes et al. (2014) constataram que a farinha de yacon é uma fonte promissora de FOS. A farinha utilizada no estudo apresentou 35,06\% de FOS em sua composição. Desta forma, foi avaliado o efeito da farinha de yacon sobre o estado nutricional de ferro e zinco, e biomarcadores de resposta imune em crianças em idade pré-escolar. Foi observado que o yacon melhorou a resposta imune intestinal, apesar de ter apresentado nenhum efeito sobre o estado nutricional de ferro e zinco.

Lobo et al. (2006) verificaram que, em ratos saudáveis alimentados com uma dieta contendo 5\% de FOS, promoveu-se uma redução do $\mathrm{pH}$ luminal, contribuindo para uma melhor absorção de cálcio e magnésio, e evidenciando o papel importante dos FOS na manutenção da saúde dos ossos.

Estudos experimentais e clínicos, observados por Caetano et al. (2016), relataram que o consumo de yacon contribui com a redução do risco de câncer de cólon, diabetes e obesidade. Os FOS do yacon modulam a microbiota intestinal humana, aumentam a absorção de glicose, estimulam a secreção de insulina pelo pâncreas e modulam as vias celulares relacionadas à homeostase lipídica. Assim, o yacon pode ser efetivamente usado com um suplemento dietético para redução dos riscos de doenças crônicas.

Genta et al. (2005) analisaram os efeitos do consumo de farinha de yacon seca como suplemento dietético para ratos. A farinha utilizada apresentou 58,3\% de FOS. Foram avaliados dois níveis diários de ingestão, 340 e $6.800 \mathrm{mg}$ de FOS/ kg de peso corporal. Em ambos os níveis, o yacon foi bem tolerado e não produziu qualquer resposta negativa, toxicidade ou efeito nutricional adverso.

De acordo com Roberfroid et al. (1998) e Tuohy et al. (2003), efeitos positivos de ingestão de FOS são conseguidos com doses entre 4 e 20 gramas/dia. Acima desse valor, efeitos indesejáveis são relatados quanto ao funcionamento do trato gastrointestinal. Para Coussement (1999), níveis de ingestão diários entre 20 e 30 gramas ocasionam o início de desconforto no indivíduo, de modo que seria recomendada dose diária de 10 gramas.

Padma Ishwarya \& Prabhasankar (2013) adicionaram FOS na forma de pó e de xarope em biscoitos e observaram que a adição de FOS diminuiu o índice glicêmico do biscoito.

De acordo com as alegações de propriedades funcionais e/ou de saúde, da ANVISA, os FOS podem ser utilizados desde que a recomendação de consumo diário do produto pronto para consumo forneça, no mínimo, 5 gramas de FOS, sendo que a porção deve fornecer, no mínimo, 2,5 gramas. O uso do ingrediente não deve 
ultrapassar 30 gramas na recomendação diária do produto pronto para consumo, conforme indicação do fabricante (Agência Nacional de Vigilânica Sanitária, 2019).

\subsection{Aspectos tecnológicos e sensoriais}

A busca por um estilo de vida mais saudável tem elevado o consumo de alimentos que sejam aceitáveis do ponto de vista sensorial e que, além disso, apresentem efeitos benéficos à saúde. Nesse contexto, a indústria de alimentos tem buscado a aplicação de compostos diferenciados e que possuam boas propriedades sensoriais e funcionais, em que os FOS têm se destacado.

Em alguns casos, os FOS são utilizados para melhorar a qualidade sensorial dos alimentos e fornecer benefícios nutricionais (Tabela 2) (Franck, 2002). Estes presentam maior solubilidade e capacidade de retenção de água que a sacarose. Além disso, não cristalizam, não precipitam e podem ser usados para alterar a temperatura de congelamento de alimentos. Por fim, os FOS apresentam baixo poder calórico (Yun, 1996; Santana \& Cardoso, 2008).

Tabela 2. Influência da adição de FOS em produtos alimentícios.

\begin{tabular}{ccc}
\hline Contribuição & Produto & Referência \\
\hline Melhoria da aceitação sensorial e perfil de textura & Mousse & Xavier-Santos et al. (2019) \\
\hline Redução da sinérese & Iogurte & Gonzalez et al. (2011) \\
\hline Poder adoçante & Chocolate & Folly et al. (2013) \\
\hline Redução do valor energético & Salsichas & Cáceres et al. (2004) \\
\hline Melhoria da doçura e do valor nutricional & Pão doce & Folly et al. (2013) \\
\hline Redução do índice glicêmico & Biscoito & Padma Ishwarya \& Prabhasankar (2013) \\
\hline Melhoria da qualidade da massa & Pão de massa congelada & Park et al. (2016) \\
\hline
\end{tabular}

Por serem altamente higroscópicos, podem ser aplicados em produtos para aumentar a umectância e diminuir a atividade de água, assegurando a estabilidade microbiológica (Franck, 2002).

Os FOS possuem um perfil adoçante semelhante ao da sacarose, mas com cerca de $35 \%$ da sua doçura. Devido a essa característica, podem substituir o açúcar parcial ou totalmente, se combinado com edulcorantes de alta intensidade, fornecendo um perfil de sabor balanceado e mascarando o sabor residual (GonzalezTomas et al., 2008). Como possuem propriedades semelhantes às da sacarose, também podem ser usados para melhorar o mouthfeel (sensação bucal) dos produtos em que são adicionados, mas sem formar cristais (Meyer et al., 2011; Tárrega et al., 2011).

Como não possuem sabores indesejáveis e não alteram a viscosidade dos produtos, diferentemente de outras fibras, possibilitam o desenvolvimento de alimentos ricos em fibras, semelhantes aos originais. A sua alta solubilidade ainda permite que ele seja utilizado em produtos lácteos; isso aumenta a ação das culturas probióticas adicionadas, exercendo efeito simbiótico (Franck, 2002; Niness, 1999).

Park et al. (2016) estudaram a adição de 3 a 9\% de FOS à massa de panificados congelada. Foi verificado que houve uma melhoria na qualidade do cozimento da massa, mesmo após o armazenamento por até oito semanas estocados a $-18{ }^{\circ} \mathrm{C}$, resultando no aumento do volume e do teor de umidade dos pães, e na redução da dureza. A adição de $6 \%$ de FOS foi a que mostrou melhor efeito sobre a qualidade da massa.

Moscatto et al. (2004) utilizaram farinha de yacon e inulina em formulação de bolo de chocolate, em que obtiveram índices de preferência de $78 \%$ para a formulação padrão (controle), $79 \%$ para a formulação contendo inulina e $73 \%$ para a formulação contendo farinha de yacon.

Ghavidel et al. (2014) avaliaram o efeito da adição de sucos mistos de maçã e laranja com FOS. Os resultados da análise sensorial mostraram que, após dois meses de armazenamento, as bebidas possuíam aceitabilidade sensorial e que, ao se aumentar a concentração de FOS, as notas para o odor aumentavam, 
porém caíam as notas para o sabor. Segundo o estudo, a combinação de açúcar e FOS poderia produzir bebidas adicionadas com um melhor sabor.

\section{Considerações finais}

O interesse na utilização dos FOS como ingredientes em formulações de alimentos tem aumentado consideravelmente nos últimos anos, especialmente devido aos efeitos benéficos à saúde, cientificamente comprovados. Entretanto, estudos ainda necessitam ser realizados avaliando a substituição parcial/total de ingredientes, como a sacarose e a gordura, em diversas classes de alimento, a fim de encontrar os níveis ótimos de substituição. Porém, o emprego de açúcares amplamente comercializados restringe a utilização do FOS, devido, principalmente, à disponibilidade e ao custo. Além disso, as formas de produção e obtenção de FOS precisam ser aprimoradas, de forma a melhorar o rendimento.

\section{Referências}

Agência Nacional de Vigilânica Sanitária - ANVISA. (2019). Alimentos com alegações de propriedades funcionais e ou de saúde. Brasília: ANVISA. Recuperado em 9 de julho de 2019, de http://www.agricultura.gov.br/assuntos/inspecao/produtosvegetal/legislacao-1/biblioteca-de-normas-vinhos-e-bebidas/alegacoes-de-propriedade-funcional-aprovadas_anvisa.pdf

Alles, M. J. L. (2012). Aplicação de processos de separação por membranas para a obtenção de frutooligossacarídeos funcionais a partir da raiz de yacon (Smallanthus sonchifolius) (Dissertação de mestrado). Universidade Federal do Rio Grande do Sul, Porto Alegre.

Antošová, M., \& Polakovič, M. (2001). Fructosyltransferases: The enzymes catalyzing production of fructooligosaccharides. Chemical Papers, 55(6), 350-358.

Blanch, M., Sanchez-Ballesta, M. T., Escribano, M. I., \& Merodio, C. (2011). Fructo-oligosaccharides in table grapes and response to storage. Food Chemistry, 129(3), 724-730. PMid:25212291.

Buddington, K. K., Donahoo, J. B., \& Buddington, R. K. (2002). Dietary oligofructose and inulin protect mice from enteric and systemic pathogens and tumor inducers. The Journal of Nutrition, 132(3), 472-477. PMid:11880573.

Cáceres, E., García, M. L., Toro, J., \& Selgas, M. D. (2004). The effect of fructooligosaccharides on the sensory characteristics of cooked sausages. Meat Science, 68(1), 87-96. PMid:22062011.

Caetano, B. F. R., De Moura, N. A., Almeida, A. P. S., Dias, M. C., Sivieri, K., \& Barbisan, L. F. (2016). Yacon (Smallanthus sonchifolius) as a food supplement: Health-promoting benefits of fructooligosaccharides. Nutrients, 8(7), PMid:27455312.

Costa, N. M. B., \& Peluzio, M. C. G. (2012). Nutrição básica e metabolismo (1. ed.). Viçosa: Editora UFV.

Courtin, C. M., Swennen, K., Verjans, P., \& Delcour, J. A. (2009). Heat and pH stability of prebiotic arabinoxylooligosaccharides, xylooligosaccharides and fructooligosaccharides. Food Chemistry, 112(4), 831-837.

Coussement, P. A. A. (1999). Nutritional and health benefits of inulin and oligofructose. The Journal of Nutrition, 129(7, Suppl.), 1402S-1406S. PMid:10447430.

Damodaran, S., Parkin, K. L., \& Fennema, O. R. (2010). Química de alimentos de Fennema (4. ed.). Porto Alegre: Artmed.

Figueroa-González, I., Quijano, G., Ramírez, G., \& Cruz-Guerrero, A. (2011). Probiotics and prebiotics-perspectives and challenges. Journal of the Science of Food and Agriculture, 91(8), 1341-1348. PMid:21445871.

Folly, G. A. F., Silva, E. N., Verner, F. V., Silva, F. C. S., \& Volp, A. C. P. (2013). Acceptance of handmade products containing nuts and fructooligosaccharides. Nutrición Hospitalaria, 28(1), 86-92. PMid:23808434.

Fortes, R. C., \& Muniz, L. B. (2009). Efeitos da suplementação dietética com frutooligossacarídeos e inulina no organismo humano: Estudo baseado em evidências. Ciência \& Saúde, 20(3), 241-252.

Franck, A. (2002). Technological functionality of inulin and oligofructose. British Journal of Nutrition, 87(Suppl. 2), 287-291. PMid:12088531.

Genta, S. B., Cabrera, W. M., Grau, A., \& Sánchez, S. S. (2005). Subchronic 4-month oral toxicity study of dried Smallanthus sonchifolius (yacon) roots as a diet supplement in rats. Food and Chemical Toxicology, 43(11), 1657-1665. PMid:15979774.

Ghavidel, R. A., Karimi, M., Davoodi, M., Jahanbani, R., \& Asl, A. F. A. (2014). Effect of fructooligosaccharide fortification on quality characteristic of some fruit juice beverages (apple \& orange juice). International Journal of Farming and Allied Sciences, 3, 141-146.

Gibson, G. R., \& Roberfroid, M. B. (1995). Dietary modulation of the human colonic microbiota-introducing the concept of prebiotics. The Journal of Nutrition, 125(6), 1401-1412. PMid:7782892.

Gonzalez, N. J., Adhikari, K., \& Sancho-Madriz, M. F. (2011). Sensory characteristics of peach-flavored yogurt drinks containing prebiotics and synbiotics. Lebensmittel-Wissenschaft + Technologie, 44(1), 158-163.

Gonzalez-Tomas, L., Coll-Marqués, J., \& Costell, E. (2008). Viscoelasticity of inulin-starch-based dairy systems. Influence of inulin average chain length. Food Hydrocolloids, 22(7), 1372-1380. 
IUB-IUPAC Joint Commission on Biochemical Nomenclature. (1982). Abbreviated terminology of oligosaccharide chains. The Journal of Biological Chemistry, 257(7), 3347-3351. PMid:7061480.

Kaczmarczyk, M. M., Miller, M. J., \& Freund, G. G. (2012). The health benefits of dietary fiber: Beyond the usual suspects of type 2 diabetes mellitus, cardiovascular disease and colon cancer. Metabolism: Clinical and Experimental, 61(8), $1058-1066$. PMid:22401879.

L'Homme, C., Puigserver, A., \& Biagini, A. (2003). Effect of food-processing on the degradation of fructooligosaccharides in fruit. Food Chemistry, 82(4), 533-537.

Li, W., Zhang, J., Yu, C., Li, Q., Dong, F., Wang, G., Gu, G., \& Guo, Z. (2015). Extraction, degree of polymerization determination and prebiotic effect evaluation of inulin from Jerusalem artichoke. Carbohydrate Polymers, 121, 315-319. PMid:25659704.

Lingyun, W., Jianhua, W., Xiaodong, Z., Da, T., Yalin, Y., Chenggang, C., Tianhua, F., \& Fan, Z. (2007). Studies on the extracting technical conditions of inulin from Jerusalem artichoke tubers. Journal of Food Engineering, 79(3), 1087-1093.

Lobo, A. R., Colli, C., \& Filisetti, T. M. C. C. (2006). Fructooligosaccharides improve bone mass and biomechanical properties in rats. Nutrition Research, 26(8), 413-420.

Lorenzoni, A. S. G., Aydos, L. F., Klein, M. P., Rodrigues, R. C., \& Hertz, P. F. (2014). Fructooligosaccharides synthesis by highly stable immobilized $\beta$-fructofuranosidase from Aspergillus aculeatus. Carbohydrate Polymers, 103(1), 193-197. PMid:24528719.

Meyer, D., Bayarri, S., Tárrega, A., \& Costell, E. (2011). Inulin as texture modifier in dairy products. Food Hydrocolloids, 25(8), 1881-1890.

Morris, C., \& Morris, G. A. (2012). The effect of inulin and fructo-oligosaccharide supplementation on the textural, rheological and sensory properties of bread and their role in weight management: A review. Food Chemistry, 133(2), 237-248. PMid:25683391.

Moscatto, J. A., Prudêncio-Ferreira, S. H., \& Hauly, M. C. O. (2004). Farinha de yacon e inulina como ingredientes na formulação de bolo de chocolate. Food Science and Technology, 24(4), 634-640.

Mussatto, S. I., \& Mancilha, I. M. (2007). Non-digestible oligosaccharides: A review. Carbohydrate Polymers, 68(3), 587-597.

Nemukula, A., Mutanda, T., Wilhelmi, B. S., \& Whiteley, C. G. (2009). Response surface methodology: Synthesis of short chain fructooligosaccharides with a fructosyltransferase from Aspergillus aculeatus. Bioresource Technology, 100(6), 2040-2045. PMid: 19028090.

Niness, K. R. (1999). Inulin and oligodructose: What are they? The Journal of Nutrition, 129(7, Suppl.), 1402-1406. PMid: 10395607.

Ordónez, J. A., Rodríguez, M. I. C., Álvarez, L. F., Sanz, M. L. G., Minguillón, G. D. G. F., Perales, L. H., \& Cortecero, M. D. S. (2005). Tecnologia de alimentos. Porto Alegre: Artmed.

Padma Ishwarya, S., \& Prabhasankar, P. (2013). Fructooligosaccharide: Retention during baking and its influence on biscuit quality. Food Bioscience, 4, 68-80.

Park, E. Y., Jang, S. B., \& Lim, S. T. (2016). Effect of fructo-oligosaccharide and isomalto-oligosaccharide addition on baking quality of frozen dough. Food Chemistry, 213, 157-162. PMid:27451167.

Passos, L. M. L., \& Park, Y. K. (2003). Frutooligossacarídeos: Implicações na saúde humana e utilização em alimentos. Ciência Rural, 33(2), 385-390.

Roberfroid, M. B., Van Loo, J. A. E., \& Gibson, G. R. (1998). The bifidogenic nature of chicory inulin and its hydrolysis products. The Journal of Nutrition, 128(1), 11-19. PMid:9430596.

Roberfroid, M. (1993). Dietary fiber, inulin, and oligofructose: A review comparing their physiological effects. Critical Reviews in Food Science and Nutrition, 33(2), 103-148. PMid:8257475.

Sangeetha, P. T., Ramesh, M. N., \& Prapulla, S. G. (2005). Maximization of fructooligosaccharide production by two stage continuous process and its scale up. Journal of Food Engineering, 68(1), 57-64.

Santana, I., \& Cardoso, M. H. (2008). Raiz tuberosa de yacon (Smallanthus sonchifolius): Potencialidade de cultivo, aspectos tecnológicos e nutricionais. Ciência Rural, 38(3), 898-905.

Shiomi, N., Abe, T., Kikuchi, H., Aritsuka, T., Takata, Y., Fukushi, E., Fukushi, Y., Kawabata, J., Ueno, K., \& Onodera, S. (2016). Structural analysis of novel kestose isomers isolated from sugar beet molasses. Carbohydrate Research, 424, 1-7. PMid:26918514.

Silva, A. S. S., Haas, P., Sartori, N. T., Anton, A. A., \& Francisco, A. (2007). Frutoligossacarídeos: Fibras alimentares ativas. Boletim do Centro de Pesquisa e Processamento de Alimentos, 25(2), 295-304.

Singh, R. S., \& Singh, R. P. (2010). Production of fructooligosaccharides from inulin by endoinulinases and their prebiotic potential. Food Technology and Biotechnology, 48(4), 435-450.

Singh, R. S., Singh, R. P., \& Kennedy, J. F. (2016). Recent insights in enzymatic synthesis of fructooligosaccharides from inulin. International Journal of Biological Macromolecules, 85, 565-572. PMid:26791586.

Tárrega, A., Torres, F. D., \& Cotell, E. (2011). Influence of the chain-length distribution of inulin on the rheology and microstructure of prebiotic dairy desserts. Journal of Food Engineering, 104(3), 356-363.

Tuohy, K. M., Probert, H. M., Smejkal, C. W., \& Gibson, G. R. (2003). Using probiotics and prebiotics to improve gut health. Drug Discovery Today, 8(15), 692-700. PMid:12927512. 
Vaz-Tostes, M., Viana, M. L., Grancieri, M., Luz, T. C., Paula, Hd., Pedrosa, R. G., \& Costa, N. M. (2014). Yacon effects in immune response and nutritional status of iron and zinc in preschool children. Nutrition, 30(6), 666-672. PMid:24631386.

Vega, R., \& Zuniga-Hansen, M. E. (2015). The effect of processing conditions on the stability of fructooligosaccharides in acidic food products. Food Chemistry, 173, 784-789. PMid:25466090.

Vega-Paulino, R. J., \& Zúniga-Hansen, M. E. (2012). Potential application of commercial enzyme preparations for industrial production of short-chain fructooligosaccharides. Journal of Molecular Catalysis. B, Enzymatic, 76, 44-51.

Voragen, A. G. J. (1998). Technological aspects of functional food-related carbohydrates. Trends in Food Science \& Technology, 9(8-9), 328-335.

Wang, J., Sun, B., Cao, Y., Tian, Y., \& Wang, C. (2009). Enzymatic preparation of wheat bran xylooligosaccharides and their stability during pasteurization and autoclave sterilization at low pH. Carbohydrate Polymers, 77(4), 816-821.

Xavier-Santos, D., Bedani, R., Perego, P., Converti, A., \& Saad, S. M. I. L. (2019). acidophilus La-5, fructo-oligosaccharides and inulin may improve sensory acceptance and texture profile of a synbiotic diet mousse. LWT, 105, 329-335.

Yun, J. W. (1996). Fructooligosaccharides-Occurrence, preparation, and application. Enzyme and Microbial Technology, 19(2), 107-117.

Zakrzewska, M. E., Bogel-Łukasik, E., \& Bogel-Łukasik, R. (2010). Solubility of carbohydrates in ionic liquids. Energy \& Fuels, 24(2), 737-745.

Financiamento: Nenhum. 